\title{
Established and novel risk factors for atrial fibrillation in women compared with men
}

Sanne A.E. Peters ${ }^{1}$ and Mark Woodward ${ }^{1,2,3,4}$

1 The George Institute for Global Health, University of Oxford, Oxford, UK

2 Cardiovascular Epidemiology Unit, Institute of Cardiovascular Research, University of Dundee, UK 3 The George Institute for Global Health, University of New South Wales, Sydney, Australia

4 Department of Epidemiology, Johns Hopkins University, Baltimore MD, USA

Correspondence to:

Dr. Sanne Peters

The George Institute for Global Health, University of Oxford Le Gros Clark Building, South Parks Road, Oxford OX1 3QX, UK

T: +44 1865617200 | F: +44 1865617202

E: sanne.peters@georgeinstitute.ox.ac.uk

Keywords: risk factors; atrial fibrillation; sex differences; cohort studies Word count: 2959 


\section{Abstract}

Background: Atrial fibrillation is a stronger risk factor for cardiovascular disease in women than men. We assessed whether there are sex differences in the effects of forty-three established and novel risk factors and the risk of incident AF.

Methods: Data were used from the Scottish Heart Health Extended Cohort, a prospective cohort study with over 20 years of follow-up for AF incidence. Cox regression models were used to obtain the adjusted sex-specific hazard ratios (HRs) and 95\% confidence intervals, and the women-to-men ratio of HRs (RHR), of incident AF associated with personal characteristics, smoking, physical measurements, diabetes mellitus, and lipids, inflammatory, cardiac, diet-related, and renal-related markers.

Results: Overall, 15,737 participants (52\% women) were included. There were sex differences in the relationship between a 1-standard deviation increase in BMI, NT-pro-BNP, uric acid, and cystatin-C and the risk of AF. The HRs were $1.17(1.08 ; 1.27)$ in women and $1.36(1.24 ; 1.49)$ in men for BMI (RHR: 0.86 [0.77; 0.97$]) ; 1.84(1.62 ; 2.09)$ in women and $1.54(1.40 ; 1.68)$ in men for NT-proBNP (RHR: $1.22[1.05 ; 1.42]) ; 1.27(1.14 ; 1.41)$ in women and $1.10(1.00 ; 1.20)$ in men for uric acid (RHR: 1.17 [1.01; 1.35]); and $1.22(1.13 ; 1.32)$ in women and $1.07(0.96 ; 1.18)$ in men for cystatin-c (RHR: 1.16 $[1.05 ; 1.27])$.

Conclusion: Higher BMI is a stronger risk factor for AF in men whereas elevated NT-pro-BNP, uric acid, and cystatin-C were more strongly associated with the risk of AF in women. 


\section{Key questions}

\section{What is already known about this subject?}

- Several factors have been associated with the risk of atrial fibrillation (AF).

- Although atrial fibrillation is a stronger risk factor for cardiovascular disease in women than men, evidence on sex differences on risk factors for the development of AF is limited.

\section{What does this study add?}

- In this prospective cohort study with over 20 years of follow-up for AF incidence, that older age, lower socioeconomic status, taller stature, and increased systolic blood pressure and CRP had a similar impact on the risk of AF in women and men

- A higher BMI was more strongly associated with the risk of AF in men than women.

- The association between elevated levels of NT-pro-BNP, uric acid, and cystatin-C and the risk of AF was stronger in women than men.

\section{How might this impact on clinical practice?}

- This study adds to the evidence of clinically meaningful sex differences in the effects of risk factors for AF, which could be taken into account in clinical guidelines, clinical decision making, and health promotion efforts to reduce the burden of AF. 


\section{Introduction}

Atrial fibrillation (AF) is an increasingly common cardiac disease that causes an irregular and often abnormally fast heart rate. In 2010, an estimated 12.6 million women and 20.9 million men had AF. ${ }^{1}$ The 2016 Global Burden of Disease study indicated that 4.0 deaths per 100,000 women and 2.5 deaths per 100,000 men were attributable to $\mathrm{AF}^{2}$ equating to a $52 \%$ increased death rate in women and a 73\% increased death rate in men since 1990.

There are important differences between women and men in the clinical presentation, prognosis, and outcomes of $A F$, which in part is explained by the earlier onset of $A F$ in men and differences in risk factor profiles. ${ }^{3,4}$ Pooled analyses of 30 studies including over 4 million participants showed that the excess risk of cardiovascular disease and death associated with AF was twice as high in women compared with men. ${ }^{5}$ Several classical risk factors for cardiovascular disease have also been associated with the risk of AF, including age, high blood pressure, excess weight, and preexisting cardiovascular disease. ${ }^{6,7}$ More recently, several inflammatory, cardiac, and renal markers have also been associated with the risk of $A F^{8-10}$ although the evidence is inconsistent and often not provided separately for women and men. However, the BiomarCaRE consortium recently demonstrated that there are sex differences in the association between some, mostly classical, risk factors and the risk of AF. ${ }^{11}$ Robust evidence on the existence of sex differences in risk factors for $A F$ would provide new insights in the sex-specific etiology of AF and could provide an impetus to take such differences into account in clinical guidelines, clinical decision making, and health promotion efforts. ${ }^{12}$

The aim of the present study was to examine the sex-specific association of forty-three established and novel risk factors and the risk of AF among women compared with men, and to quantify potential sex differences, in the Scottish Heart Health Extended Cohort, a prospective cohort study with over 20 years of follow-up for AF incidence.

\section{Methods}

Study population, risk factor assessment, and follow-up

SHHEC includes men and women men and women from multiple overlapping studies. The Scottish Heart Health Study randomly recruited participants aged 40 to 59 years across 23 districts of Scotland from 1984 to $1987 .{ }^{13}$ The Scottish MONICA project recruited participants aged 25 to 64 years in Edinburgh and north Glasgow in 1986, and north Glasgow again in 1989, 1992 (ages 25 to 75 years), and $1995 .{ }^{14}$ All participants provided written informed consent and completed a questionnaire which solicited information on demographic and lifestyle factors and past medical history. They attended a recruitment survey clinic where physical measurements and blood samples were taken using standardised procedures. Detailed information on smoking and its biochemistry 
were obtained, which included measurement of expired carbon monoxide (CO), serum thiocyanate, and serum cotinine. Participants were included in the analyses if they were aged 30-74 years at recruitment and did not have a history of cardiovascular disease at study baseline. Previously diagnosed cardiovascular disease was identified by self-report and hospital discharge diagnoses: any coronary heart disease; cerebrovascular disease, including transient ischaemic attacks; heart failure; and peripheral arterial disease. No specific question on the presence of pre-existing of AF was solicited. Incident AF was defined as the presence of International Classification of Diseases 9 (ICD-9) code 427.31 in any of the multiple cause fields of hospital diagnoses or on the death certificate. Follow-up ended on December 31, 2009, or upon the first fatal or non-fatal diagnosis of AF, for all participants. Ethical approval was received from all relevant medical research ethics committees covering the individual populations involved.

\section{Statistical analyses}

Baseline characteristics are presented as means (standard deviation) for continuous variables and as percentages for categorical variables, separately for women and men. Cox proportional hazards models were used to obtain the sex-specific hazard ratios (HRs) and 95\% confidence intervals ( $\mathrm{Cls}$ ) for AF associated with each risk factor. The women-to-men ratio of HRs were derived from the interaction term between sex and each risk factor included in the model. Models were first adjusted for age only and then also for the other risk factors included in the ASSIGN score, ${ }^{15}$ except sex. These are family history of coronary heart disease (CHD), socioeconomic status (based on the postcodederived Scottish Index of Multiple Deprivation), cigarette equivalent dose (incorporating selfreported cigarette consumption, the equivalent for pipe smokers, and smoking deceivers using their biochemical results), systolic blood pressure, total cholesterol, and high-density (HDL) cholesterol. The Cox proportional hazards assumption was assessed using scaled Schoenfeld residuals and there was no evidence that the proportionality assumption was unreasonable. For all analyses, multiple imputation using chained equations by predictive mean matching with 5 imputation datasets was used to impute missing values in the included risk factors. The imputation model for each risk factor included all other risk factors and (time to) the study outcome. Statistical analyses were conducted using R version 3.3.0 (R Foundation, Vienna, Austria).

\section{Results}

Overall, 8,185 women and 7,552 men were included. The mean age at recruitment was 49 years and $40 \%$ of women and $52 \%$ of men were current smokers. The mean body mass index was $26 \mathrm{~kg} / \mathrm{m}^{2}$ in both sexes and the mean systolic BP was $131 \mathrm{mmHg}$ in women and $134 \mathrm{mmHg}$ in men. During a median follow-up of 22.6 years, 1,036 (43\% women) incident cases of AF were recorded. Age- 
adjusted HRs and the ratios of HRs are shown in Table 2 and the corresponding multiple-adjusted results are shown in Figures 1-3.

\section{Personal characteristics}

Older age and increases in the SIMD score for socioeconomic deprivation were each associated with a higher risk of $A F$ in both sexes. A family history of CHD was associated with the risk of AF in men, but not women. There was no evidence for sex differences in the association between personal characteristics and the risk of AF.

\section{Smoking}

In the age-adjusted models, current smoking and increases in expired air $\mathrm{CO}$ and cotinine were associated with an increased risk of AF in women, whereas all smoking-related variables were associated with an increased risk of $A F$ in men. Following multiple adjustment, only increases in the cigarette equivalent dose remained associated with an elevated risk of AF in men (HR per SD increase $1.14[1.06 ; 1.23])$, but the difference with women was not statistically significant.

\section{Physical measurements}

Taller stature and increases in weight, BMI, and waist circumference were each associated with a higher risk of $\mathrm{AF}$ in both sexes. $\mathrm{BMI}$ was a stronger risk factor for $\mathrm{AF}$ in men than in women; the multiple-adjusted HRs per SD increase in BMI were $1.17(1.08 ; 1.27)$ in women and $1.36(1.24 ; 1.49)$ in men. The corresponding women-to-men ratio of HRs was $0.86(0.77 ; 0.97)$. The excess risk of AF associated with increases in systolic blood pressure was similar between the sexes; the multipleadjusted HRs per SD increase in systolic BP were $1.25(1.15 ; 1.36)$ in women and $1.23(1.14 ; 1.32)$ in men.

\section{Diabetes mellitus}

A prior history of diabetes mellitus was associated with the risk of AF in women in the age-adjusted, but not in the multiple-adjusted analyses. In men, increased levels of non-fasting glucose were marginally associated with the risk of AF in the age-adjusted, but not in the multiple-adjusted, models.

\section{Lipids}

The age-adjusted analyses provided some suggestion that increased levels of triglycerides in women and increased levels of lipoprotein (a) in men were associated with a higher risk of AF. However, in the multiple-adjusted models, none of the lipids evaluated were associated with the risk of AF in either sex. 
Inflammatory markers

C-reactive protein was associated with an increased risk of $A F$ in both sexes; the multiple-adjusted HRs associated with each 1-SD increase in CRP were $1.13(1.02 ; 1.26)$ in women and $1.16(1.05 ; 1.27)$ in men. Elevated fibrinogen was associated with an increased risk of AF in men only.

\section{Cardiac markers}

Elevated NT-pro-BNP, but not hsTroponin I, was significantly associated with the risk of AF in both women and men; the multiple-adjusted HRs per SD increase in NT-pro-BNP were $1.84(1.62 ; 2.09)$ in women and $1.54(1.40 ; 1.68)$ in men. The corresponding women-to-men ratio of HRs was $1.22(1.05$; 1.42).

\section{Diet-related markers}

Diet-related markers were not associated with the risk of AF, except for elevated $\gamma$-glutamyl transferase which, after multiple adjustment, was marginally related to an increased risk of AF in men $(1.10[1.01 ; 1.20])$, but not women.

\section{Renal-related markers}

Uric acid was significantly related to an increased risk of $A F$, with some evidence to suggest that the magnitude of the association was greater in women than men. The multiple-adjusted HRs were 1.27 $(1.14 ; 1.41)$ in women and $1.10(1.00 ; 1.20)$ in men; the corresponding women-to-men ratio of HRs was 1.17 (1.01; 1.35). Creatinine was not associated with an increased risk of AF in either sex, whereas elevated levels of cystatin-c were associated with the risk of AF in women but not men. The multiple-adjusted HRs of AF associated with a 1-SD increase in cystatin-c were $1.22(1.13 ; 1.32)$ in women and $1.07(0.96 ; 1.18)$ in men; the corresponding women-to-men ratio was $1.16(1.05 ; 1.27)$.

\section{Discussion}

This large cohort study with over 20 years of prospective follow-up demonstrated that there are sex differences in the magnitude of the association between several established and novel risk factors and the risk of AF. A higher BMI was more strongly associated with the risk of AF in men than women. In contrast, the association between elevated levels of NT-pro-BNP, uric acid, and cystatin-C and the risk of $\mathrm{AF}$ was stronger in women than men.

The findings from this study expands prior research on sex differences in risk factors for AF. Elevated $\mathrm{BMI}$ is a strong risk factor for AF and several, but not all, studies have suggested that the strength of the association may be stronger in men than women. ${ }^{11,16-18}$ The BiomarCaRE Consortium 
among nearly 80,000 individuals and over 4,000 cases of incident AF demonstrated that a 1-SD (4.67 $\mathrm{kg} / \mathrm{m}^{2)}$ increase in BMI was associated with a $31 \%$ higher risk of AF in men, compared with a $18 \%$ higher risk in women. The corresponding $11 \%$ greater excess risk of AF associated with increases in $\mathrm{BMI}$ in men reported in BiomarCaRE is almost identical to the $14 \%$ greater excess risk found in the present study. Although there was no statistical evidence for a sex difference, the Framingham Heart Study of 4,750 individuals and 457 AF events also reported that a 1-SD increase in BMI was associated with a $27 \%$ higher risk of $\mathrm{AF}$ in men and a $9 \%$ higher risk of $\mathrm{AF}$ in women. ${ }^{18}$ Similarly, the Busselton health study of 4,200 individuals reported that a 1-SD increase in BMI was associated with an increased risk of AF of $64 \%$ in men and $16 \%$ in women. ${ }^{16}$ In the Malmö diet and cancer study of 30,000 individuals, obesity was also a stronger risk factor for AF in men than women, but the difference was not statistically significant. ${ }^{17}$ In contrast, a meta-analysis of three population-based cohort studies involving 68,000 individuals showed that the excess risks of AF conferred by overweight and obesity was similar between the sexes, but with a somewhat larger effect in obese women than in obese men. ${ }^{19}$ Height, measures of central obesity, and measures of body impedance, such as body fat mass, body fat percentage, and lean body mass, have also been associated with the risk of AF. ${ }^{20,21}$ Data from the Women's Health Initiative suggested that the risk of AF conferred by higher BMI was primarily driven by the association between lean body mass and AF. ${ }^{20}$ Furthermore, the Danish Diet, Cancer and Health Study reported that lean body mass was the predominant anthropometric risk factor for AF. ${ }^{21}$ After adjustment for lean body mass, none of the obesity-related anthropometric measures was associated with the risk of AF. Sex differences in body fat distribution and body composition are well-established, with a predominance of subcutaneous fat and a lower lean body mass in women and more visceral fat and lower fat percentage in men. ${ }^{22,23}$ Despite these differences, the association between higher lean body mass and the risk of AF does not differ between women and men. ${ }^{21}$ Similarly, in the present study, we found that the association of height, waist-to-hip ratio, and waist circumference with the risk of AF was similar in women and men. In contrast, our prior analyses on risk factors for CHD in the UK Biobank found that measures of central adiposity, but not BMI, were more strongly associated with the risk of $\mathrm{CHD}$ in women than in men. ${ }^{24}$ Further research is needed to determine understand how sex differences in body fat distribution and composition might have a differential impact on the risk of AF, and how and why this may differ from other cardiovascular phenotypes.

The study provides novel evidence for the presence of sex differences in the relationship between the biomarkers NT-pro-BNP, uric acid, and cystatin-C and the risk of AF, each with stronger associations in women than men. Indeed, overall the discrimination of AF events, using all the covariates we analysed, was higher in women than men: c-statistics were $0.81(0.78 ; 0.84)$ and 0.78 $(0.75 ; 0.80)$. Although there is an abundance of literature to support the relationship between NT- 
pro-BNP and the risk of AF, sex-specific effects have only sparsely been reported. ${ }^{8,9,25}$ Recently, the BiomarCaRE consortium showed that increases in NT-pro-BNP conferred an eleven percent greater excess risk of AF in women than men. ${ }^{11}$ Although this sex difference was not statistically significant in BiomarCaRE, it is directionally consistent with the $22 \%$ greater excess risk of AF in women than men associated with NT-pro-BNP reported in this study.

Uric acid is a surrogate marker of oxidative stress and has been linked to cardiovascular disease in several studies. The sex-specific role of uric acid in the etiology of AF has been investigated in few prospective studies. The Atherosclerosis Risk in Communities (ARIC) Study among more than 15,000 participants found evidence for a stronger effect in women than men; each 1-SD increase in uric acid was associated with an $25 \%$ increased risk of AF in women, with no statistically significant association in men $(p$ for interaction $=0.01) .{ }^{26}$ In contrast, while the Troms $\varnothing$ Study of over 6,000 women and men reported that a 1-SD increase in uric was associated with an increased risk of AF of $17 \%$ in men and $40 \%$ in women, the $p$-value for interaction by sex was not statistically significant. ${ }^{10}$ Moreover, a large study in Taiwan including over 120,000 individuals showed that the relationship between hyperuricemia and new-onset AF was similar between the sexes. ${ }^{27}$ Serum uric acid levels are similar in boys and girls during childhood, but are higher in men than in women throughout adult life. Although levels of uric acid in women increase after menopause, they remain constant or decline slightly with ageing in men. The incidence of AF increases with age and it has been suggested that age-related sex differences in uric acid levels might influence the observed sex differences in the association between uric acid and the risk of $A F .{ }^{28}$ Nevertheless, relative risk estimates are typically adjusted for age and several other potential confounders, implying that age-related sex differences in uric acid levels are unlikely to account for the observed sex differences.

Cystatin-C, together with other markers of renal function, has been associated with the risk of cardiovascular disease in several studies. ${ }^{9}$ The relationship between renal function and the risk of AF, however, is considerably less well-established. Findings from the Cardiovascular Health Study indicated that cystatin-C was associated with prevalent, but not with incident, AF. ${ }^{29}$ These findings suggest that renal dysfunction and AF may share a common pathology, not fully accounted for in the multivariable analyses. On contrary, meta-analyses of nine prospective studies found that the presence of CKD was associated with an increased risk for new-onset AF of $50 \% .{ }^{30}$ Sex-specific estimates were not provided, so further research is needed to investigate whether the sex differences reported in this study are causal and to identify the mechanisms involved.

The identification and quantification of sex differences in the relationship between classic and novel risk factors and the risk of AF is important for several reasons. First, it could provide novel insights in the etiology of AF and might reveal new therapeutic targets, which may be sex-specific. Second, current and projected estimates for the burden of AF assume that the risks associated with 
certain risk factors are equivalent between the sexes. However, if this assumption is proven to be invalid, estimates should be updated to ensure that decisions regarding the allocation of health care resources are made as accurate as possible, using the best available data. Such estimates can also be used in clinical guidelines, which currently include limited information on sex differences in AF. ${ }^{12}$ Third, current risk scores for the prediction of AF neither include sex as a predictor nor use sexspecific weights for the variables included in the risk score. ${ }^{8,18} \mathrm{BMI}$ is included in the Framingham AF score and the CHARGE-AF Augmented Score includes NT-pro-BNP. ${ }^{8,18}$ In this study, we found evidence for a sex difference in each of these risk factors. If confirmed, not accounting for sex differences in the associations between these risk factors and the risk of AF could lead to inaccurate risk estimates and suboptimal treatment decisions and outcomes.

The strengths of this study include its prospective design, long-term follow-up, and measurement of a wide range of classic and novel risk factors. This allowed for a detailed assessment of sex differences in the relationship between these risk factors and the risk of AF in later life. This study also has some limitations. First, risk factor information was only collected once at recruitment into the study and we were unable to either assess or account for the impact of changes in risk factor levels during follow-up on the study findings. Second, while we adjusted our analyses for several factors, our findings are subject to residual confounding. We, however, assume that the impact of such confounding factors on the results has been similar between women and men and has not affected the validity of the sex comparisons. Third, although we excluded individuals with previous CVD, we did not have specific information on the presence of pre-existing AF. Also, the identification of incident AF cases was derived from hospital diagnoses and death certificates, which may have led to misclassification of AF incidence, especially among those with intermittent AF. We are not aware of sex differences in the misclassification of $\mathrm{AF}$, although we cannot exclude the possibility. Fourth, a substantial number of comparisons was conducted in this study and we cannot exclude the possibility that some of the reported results were due to chance. In interpreting the findings, the effect size and clinical implications should be considered alongside the confidence intervals.

In conclusion, the relationships between older age, lower socioeconomic status, taller stature, and increased systolic blood pressure and CRP and the risk of AF were largely similar between women and men. In contrast, higher BMI is a stronger risk factor for AF in men whereas elevated NT-pro-BNP, uric acid, and cystatin-C were more strongly associated with the risk of AF in women. 
Page 11 of 19 
Funding: The Scottish Heart Health Extended Cohort (SHHEC) was funded by the Scottish Health Department Chief Scientist Organization, the British Heart Foundation, and the FP Fleming Trust. The MORGAM collaboration was funded by the European Commission Seventh Framework Programme, references FP7/2007-2013 (HEALTH-F4-2007-2014113, ENGAGE; HEALTH-F3-2010-242244, CHANCES). The MORGAM Biomarker Study (serum biomarkers in the MORGAM populations) was funded by the Medical Research Council, London (reference G0601463, No 80983). The BiomarCaRE Project (Biomarkers for Cardiovascular Risk Assessment in Europe) was funded by the European Commission Seventh Framework Programme FP7/2007-2013 (reference HEALTHF2-2011-278913). SP is supported by a UK Medical Research Council Skills Development Fellowship (MR/P014550/1). Funding bodies had no role in the planning of the study, analyses, interpretation, writing or publication of the manuscript, or recruitment of participants.

Disclosures: none

Acknowledgement: The authors would like to thank Professor Hugh Tunstall-Pedoe for his comments on an earlier version of this article.

Licence: The Corresponding Author has the right to grant on behalf of all authors and does grant on behalf of all authors, an exclusive licence on a worldwide basis to the BMJ Publishing Group Ltd and its Licensees to permit this article to be published in HEART editions and any other BMJPGL products to exploit all subsidiary rights. 


\section{References}

1. Chugh SS, Havmoeller R, Narayanan K, et al. Worldwide epidemiology of atrial fibrillation: a Global Burden of Disease 2010 Study. Circulation 2014; 129(8): 837-47.

2. Institute for Health Metrics and Evaluation. GBD Compare Data Visualization. 2016. http://vizhub.healthdata.org/gbd-compare.

3. Dagres N, Nieuwlaat R, Vardas PE, et al. Gender-related differences in presentation, treatment, and outcome of patients with atrial fibrillation in Europe: a report from the Euro Heart Survey on Atrial Fibrillation. Journal of the American College of Cardiology 2007; 49(5): 572-7.

4. Lip GY, Laroche C, Boriani G, et al. Sex-related differences in presentation, treatment, and outcome of patients with atrial fibrillation in Europe: a report from the Euro Observational Research Programme Pilot survey on Atrial Fibrillation. Europace : European pacing, arrhythmias, and cardiac electrophysiology : journal of the working groups on cardiac pacing, arrhythmias, and cardiac cellular electrophysiology of the European Society of Cardiology 2015; 17(1): 24-31.

5. Emdin CA, Wong CX, Hsiao AJ, et al. Atrial fibrillation as risk factor for cardiovascular disease and death in women compared with men: systematic review and meta-analysis of cohort studies. BMJ (Clinical research ed) 2016; 532: h7013.

6. Schnabel RB, Yin X, Gona P, et al. 50 year trends in atrial fibrillation prevalence, incidence, risk factors, and mortality in the Framingham Heart Study: a cohort study. Lancet (London, England) 2015; 386(9989): 154-62.

7. Allan V, Honarbakhsh S, Casas JP, et al. Are cardiovascular risk factors also associated with the incidence of atrial fibrillation? A systematic review and field synopsis of 23 factors in 32 population-based cohorts of 20 million participants. Thrombosis and haemostasis 2017; 117(5): 83750.

8. Sinner MF, Stepas KA, Moser CB, et al. B-type natriuretic peptide and C-reactive protein in the prediction of atrial fibrillation risk: the CHARGE-AF Consortium of community-based cohort studies. Europace : European pacing, arrhythmias, and cardiac electrophysiology : journal of the working groups on cardiac pacing, arrhythmias, and cardiac cellular electrophysiology of the European Society of Cardiology 2014; 16(10): 1426-33.

9. Hijazi Z, Oldgren J, Siegbahn A, Granger CB, Wallentin L. Biomarkers in atrial fibrillation: a clinical review. European heart journal 2013; 34(20): 1475-80.

10. Tamariz L, Hernandez F, Bush A, Palacio A, Hare JM. Association between serum uric acid and atrial fibrillation: a systematic review and meta-analysis. Heart rhythm 2014; 11(7): 1102-8.

11. Magnussen C, Niiranen TJ, Ojeda FM, et al. Sex Differences and Similarities in Atrial Fibrillation Epidemiology, Risk Factors, and Mortality in Community Cohorts: Results From the BiomarCaRE Consortium (Biomarker for Cardiovascular Risk Assessment in Europe). Circulation 2017; 136(17): 1588-97.

12. Gorenek B, Pelliccia A, Benjamin EJ, et al. European Heart Rhythm Association (EHRA)/European Association of Cardiovascular Prevention and Rehabilitation (EACPR) position paper on how to prevent atrial fibrillation endorsed by the Heart Rhythm Society (HRS) and Asia Pacific Heart Rhythm Society (APHRS). Europace : European pacing, arrhythmias, and cardiac electrophysiology : journal of the working groups on cardiac pacing, arrhythmias, and cardiac cellular electrophysiology of the European Society of Cardiology 2017; 19(2): 190-225.

13. Smith WC, Crombie IK, Tavendale R, Irving JM, Kenicer MB, Tunstall Pedoe H. The Scottish Heart Health Study: objectives and development of methods. Health bulletin 1987; 45(4): 211-7.

14. Tunstall Pedoe H, Kuulasmaa K, Tolonen H, Davidson M, Mendis S, for the WHO MONICA Project. MONICA Monograph and Multimedia Sourcebook. Geneva, Switzerland: World Health Organization; 2003.

15. Woodward M, Brindle $P$, Tunstall-Pedoe H. Adding social deprivation and family history to cardiovascular risk assessment: the ASSIGN score from the Scottish Heart Health Extended Cohort (SHHEC). Heart (British Cardiac Society) 2007; 93(2): 172-6. 
16. Knuiman M, Briffa T, Divitini M, et al. A cohort study examination of established and emerging risk factors for atrial fibrillation: the Busselton Health Study. European journal of epidemiology 2014; 29(3): 181-90.

17. Smith JG, Platonov PG, Hedblad B, Engstrom G, Melander O. Atrial fibrillation in the Malmo Diet and Cancer study: a study of occurrence, risk factors and diagnostic validity. European journal of epidemiology 2010; 25(2): 95-102.

18. Schnabel RB, Sullivan LM, Levy D, et al. Development of a risk score for atrial fibrillation (Framingham Heart Study): a community-based cohort study. Lancet (London, England) 2009; 373(9665): 739-45.

19. Wanahita N, Messerli FH, Bangalore S, Gami AS, Somers VK, Steinberg JS. Atrial fibrillation and obesity--results of a meta-analysis. American heart journal 2008; 155(2): 310-5.

20. Azarbal F, Stefanick ML, Assimes TL, et al. Lean body mass and risk of incident atrial fibrillation in post-menopausal women. European heart journal 2016; 37(20): 1606-13.

21. Fenger-Gron M, Overvad K, Tjonneland A, Frost L. Lean Body Mass Is the Predominant Anthropometric Risk Factor for Atrial Fibrillation. Journal of the American College of Cardiology 2017; 69(20): 2488-97.

22. Fuente-Martin E, Argente-Arizon P, Ros P, Argente J, Chowen JA. Sex differences in adipose tissue: It is not only a question of quantity and distribution. Adipocyte 2013; 2(3): 128-34.

23. Valencak TG, Osterrieder A, Schulz TJ. Sex matters: The effects of biological sex on adipose tissue biology and energy metabolism. Redox biology 2017; 12: 806-13.

24. Peters SAE, Bots SH, Woodward M. Sex Differences in the Association Between Measures of General and Central Adiposity and the Risk of Myocardial Infarction: Results From the UK Biobank. Journal of the American Heart Association 2018; 7(5).

25. Smith JG, Newton-Cheh C, Almgren P, et al. Assessment of conventional cardiovascular risk factors and multiple biomarkers for the prediction of incident heart failure and atrial fibrillation. Journal of the American College of Cardiology 2010; 56(21): 1712-9.

26. Tamariz L, Agarwal S, Soliman EZ, et al. Association of serum uric acid with incident atrial fibrillation (from the Atherosclerosis Risk in Communities [ARIC] study). The American journal of cardiology 2011; 108(9): 1272-6.

27. Chao TF, Hung $\mathrm{CL}$, Chen SJ, et al. The association between hyperuricemia, left atrial size and new-onset atrial fibrillation. International journal of cardiology 2013; 168(4): 4027-32.

28. Nyrnes A, Toft I, Njolstad I, et al. Uric acid is associated with future atrial fibrillation: an 11year follow-up of 6308 men and women--the Tromso Study. Europace : European pacing, arrhythmias, and cardiac electrophysiology : journal of the working groups on cardiac pacing, arrhythmias, and cardiac cellular electrophysiology of the European Society of Cardiology 2014; 16(3): 320-6.

29. Deo R, Katz R, Kestenbaum B, et al. Impaired kidney function and atrial fibrillation in elderly subjects. Journal of cardiac failure 2010; 16(1): 55-60.

30. Shang W, Li L, Huang S, et al. Chronic Kidney Disease and the Risk of New-Onset Atrial Fibrillation: A Meta-Analysis of Prospective Cohort Studies. PloS one 2016; 11(5): e0155581. 
Figure legend

Figure 1: Multiple-adjusted hazard ratios, with $95 \%$ confidence intervals, of the association between individual risk factors and the risk of AF in women

Figure 2: Multiple-adjusted hazard ratios, with $95 \%$ confidence intervals, of the association between individual risk factors and the risk of $A F$ in men

Figure 3: Multiple-adjusted women-to-men ratio of HRs, with $95 \%$ confidence intervals, of the association between individual risk factors and the risk of AF 
Table 1: Baseline characteristics of study participants by sex

\section{Number of participants}

Personal

Aget

Years of education

Family history of $\mathrm{CHD},+\%$

SIMD-socioeconomic scoret

ASSIGN cardiovascular risk score

\section{Smoking}

Current tobacco smoker, \%

Cigarettes equivalent, + cigs/d

Expired carbon monoxide, ppm

Thiocyanate, Imol/L*

Cotinine, $\mathrm{ng} / \mathrm{mL}$

\section{Physical measurements}

Height, $m$

Weight, $k g$

Body mass index, $\mathrm{kg} / \mathrm{m}^{2}$

Waist circumference, $\mathrm{cm}$

Waist-to-hip ratio

Systolic $B P,+m m H g$

Diastolic $B P, \mathrm{mmHg}$

Pulse pressure, $\mathrm{mmHg}$

Pulse rate, bpm

\section{Diabetes mellitus}

Diabetes mellitus, $\uparrow \%$

Glucose, mmol/L (nonfasting)

Insulin, $\mathrm{IU} / \mathrm{mL}$ (nonfasting)

C-Peptide, $\mathrm{mg} / \mathrm{mL}^{*}$

\section{Values available}

Lipids

Total cholesterol, $+\mathrm{mmol} / \mathrm{L}$

HDL-cholesterol, $+\mathrm{mmol} / \mathrm{L}$

Triglycerides, $\mathrm{mmol} / \mathrm{L}^{*}$

LDL-cholesterol, $\mathrm{mmol} / \mathrm{L}$

Apolipoprotein A, $g / L$

Apolipoprotein $B, g / L$

Lipoprotein (a), $m g / d L^{*}$
15737

12837

15737

15737

15396

15737

15737

13444

15737

15737

15257

13738

10984

15729

15727

15725

4061

4061

15729

15729

15729

15661

12281

12365

Women

Men

8185

7552

$\begin{array}{cc}49.0(8.3) & 49.0(8.3) \\ 11.1(2.5) & 11.2(2.8) \\ 31.8 & 25.5 \\ 28.6(22.0) & 28.0(21.9) \\ 9.5(9.9) & 14.8(10.6)\end{array}$

40.2

51.6

$$
6.6(9.6)
$$

9.1 (11.9)

9.0 (11.5)

10.6 (12.5)

58.4 (1.98)

57.8 (1.99)

103 (152)

128 (171)

$\begin{array}{cc}1.60(0.06) & 1.73(0.07) \\ 65.9(12.6) & 77.9(12.5) \\ 25.8(4.8) & 26.0(3.7) \\ 80.5(12.2) & 92.2(11.6) \\ 0.79(0.071) & 0.92(0.078) \\ 130.5(21.1) & 134.0(19.4) \\ 79.6(11.9) & 83.4(12.0) \\ 50.8(15.1) & 50.6(14.1) \\ 78.6(12.3) & 76.5(13.3)\end{array}$

1.4

1.6

4.92 (1.36)

5.17 (1.54)

8.52 (9.60)

$11.2(13.2)$

2.14 (1.82)

2.41 (1.92)

14172

13460

$6.40(1.30)$

6.23 (1.16)

14158

$1.63(0.43)$

1.35 (0.37)

1.41 (1.69)

1.91 (1.77)

10833

2.75 (1.19)

2.65 (1.27)

12867

$1.64(0.30)$

$1.49(0.26)$

12866

$1.13(0.32)$

1.18 (0.30)

12773

Inflammatory 
hsC-reactive protein, $\mathrm{mg} / \mathrm{L}^{*}$

Fibrinogen, $g / L$

Homocysteine, Imol/L

Ferritin, Imol/L*

\section{Cardiac}

hsTroponin I, $p g / \mathrm{mL}^{*}$

NT-pro-BNP, pg/mL*

\section{Diet related}

25-Hydroxyvitamin D raw, $\mathrm{nmol} / \mathrm{L}$ 25-Hydroxyvitamin D adj, $n$ mol/L Vitamin B12, $\mathrm{pg} / \mathrm{mL}$

$\gamma$-Glutamyl transferase, units/L*

Renal related

Uric acid, $\mathrm{mmol} / \mathrm{L}$

Creatinine, $\mathrm{mmol} / \mathrm{L}$

Cystatin-C, $\mathrm{mg} / \mathrm{L}$
14178

11837

12847

13101

12843

12319

11527

10507

11603

11603

12355

12835

12868
$1.27(3.10) \quad 1.53(2.99)$

$2.86(0.81) \quad 2.75(0.80)$

$14.0(5.7) \quad 16.0(6.6)$

$38.0(2.97) \quad 103.0(2.64)$

$2.25(3.80) \quad 3.76(2.60)$

$61.5(2.45) \quad 31.7(2.94)$

$39.2(19.7) \quad 44.3(22.7)$

$39.4(17.9) \quad 44.0(19.3)$

$423(191) \quad 425(180)$

18.5 (1.79) $\quad 31.4$ (1.92)

$243(62) \quad 320(66)$

$81.4(11.0) \quad 95.9(12.9)$

$0.716(0.165) \quad 0.760(0.143)$

Values are means (standard deviation) for continuous variables and as percentages for categorical variables 
Table 2: Age-adjusted hazard ratios and women-to- men ratio of HRs, with $95 \%$ confidence intervals, of the association between individual risk factors and the risk of AF, by sex

\section{Personal \\ Age \\ Years of education \\ Family history of CHD \\ SIMD score \\ ASSIGN score \\ Smoking \\ Cigarette Smoker \\ Cigs equivalent/day \\ Expired air $\mathrm{CO}$ \\ Thiocyanate \\ Cotinine}

\section{Physical measurements}

Height

Weight

Body Mass Index

Waist circumference

Waist-to-hip ratio

Systolic BP

Diastolic BP

Pulse pressure

Pulse rate

\section{Diabetes mellitus}

Self-reported diabetes

Non-fasting glucose

Insulin

C-Peptide

\section{Lipids}

Total cholesterol

HDL cholesterol

LDL cholesterol

Triglycerides

Apolipoprotein A

Apolipoprotein $B$

Lipoprotein (a)

Inflammatory

C-Reactive Protein
Women Men $\quad$ Ratio of HRs

$\begin{array}{lll}2.69(2.41 ; 2.99) & 2.32(2.11 ; 2.54) & 1.16(1.01 ; 1.33) \\ 0.98(0.88 ; 1.08) & 1.00(0.93 ; 1.09) & 0.97(0.86 ; 1.11) \\ 1.19(0.98 ; 1.44) & 1.30(1.09 ; 1.56) & 0.91(0.70 ; 1.19) \\ 1.16(1.06 ; 1.28) & 1.13(1.04 ; 1.23) & 1.03(0.91 ; 1.17) \\ 1.29(1.20 ; 1.40) & 1.25(1.16 ; 1.34) & 1.04(0.95 ; 1.14)\end{array}$

$1.24(1.02 ; 1.50) \quad 1.28(1.09 ; 1.51) \quad 0.97(0.75 ; 1.24)$

$1.10(0.98 ; 1.23) \quad 1.16(1.08 ; 1.25) \quad 0.94(0.83 ; 1.08)$

$1.12(1.01 ; 1.23) \quad 1.14(1.06 ; 1.23) \quad 0.98(0.87 ; 1.11)$

$1.09(0.99 ; 1.20) \quad 1.15(1.06 ; 1.26) \quad 0.95(0.83 ; 1.08)$

$1.14(1.02 ; 1.28) \quad 1.13(1.05 ; 1.23) \quad 1.01(0.89 ; 1.15)$

$\begin{array}{lll}1.37(1.19 ; 1.57) & 1.31(1.18 ; 1.47) & 1.04(0.87 ; 1.24) \\ 1.39(1.26 ; 1.52) & 1.50(1.37 ; 1.63) & 0.93(0.82 ; 1.05) \\ 1.23(1.14 ; 1.33) & 1.41(1.29 ; 1.54) & 0.87(0.77 ; 0.98) \\ 1.45(1.30 ; 1.62) & 1.53(1.36 ; 1.72) & 0.95(0.82 ; 1.09) \\ 1.19(0.92 ; 1.54) & 1.19(1.05 ; 1.35) & 1.00(0.77 ; 1.30) \\ 1.26(1.15 ; 1.37) & 1.23(1.14 ; 1.32) & 1.02(0.92 ; 1.14) \\ 1.21(1.10 ; 1.33) & 1.16(1.07 ; 1.25) & 1.05(0.93 ; 1.19) \\ 1.37(1.18 ; 1.58) & 1.33(1.17 ; 1.52) & 1.03(0.85 ; 1.24) \\ 0.87(0.79 ; 0.96) & 0.97(0.90 ; 1.05) & 0.89(0.79 ; 1.02)\end{array}$

$1.97(1.08 ; 3.59) \quad 1.28(0.69 ; 2.40) \quad 1.54(0.65 ; 3.65)$

$1.08(0.99 ; 1.18) \quad 1.07(1.00 ; 1.15) \quad 1.01(0.90 ; 1.12)$

$1.04(0.94 ; 1.15) \quad 1.06(0.99 ; 1.14) \quad 0.98(0.87 ; 1.10)$

$1.06(0.94 ; 1.20) \quad 1.01(0.92 ; 1.12) \quad 1.05(0.90 ; 1.22)$

$\begin{array}{lll}0.98(0.89 ; 1.08) & 0.96(0.87 ; 1.07) & 1.02(0.88 ; 1.18) \\ 0.85(0.76 ; 0.95) & 0.91(0.82 ; 1.01) & 0.93(0.81 ; 1.08) \\ 1.01(0.90 ; 1.12) & 1.02(0.94 ; 1.11) & 0.99(0.86 ; 1.13) \\ 1.11(1.00 ; 1.23) & 1.04(0.95 ; 1.14) & 1.06(0.93 ; 1.23) \\ 0.90(0.79 ; 1.04) & 0.97(0.88 ; 1.06) & 0.93(0.79 ; 1.10) \\ 1.06(0.97 ; 1.17) & 1.03(0.94 ; 1.14) & 1.03(0.89 ; 1.19) \\ 1.08(0.94 ; 1.24) & 1.12(1.02 ; 1.23) & 0.97(0.83 ; 1.13) \\ & & \\ & & \\ 1.25(1.13 ; 1.39) & 1.23(1.12 ; 1.36) & 1.01(0.89 ; 1.16)\end{array}$ 
Fibrinogen

Homocysteine

Ferritin

\section{Cardiac}

hsTroponin I

NT-pro-BNP

Diet-related

25-Hydroxyvitamin D raw

25-Hydroxyvitamin D adjusted

Vitamin $B_{12}$

$\gamma$-Glutamyl transferase

\section{Renal-related}

Uric Acid

Creatinine

Cystatin-C
$1.10(0.99 ; 1.23) \quad 1.16(1.07 ; 1.25) \quad 0.95(0.84 ; 1.08)$

$1.09(0.98 ; 1.22) \quad 1.05(0.96 ; 1.13) \quad 1.04(0.92 ; 1.19)$

$1.06(0.94 ; 1.20) \quad 1.03(0.92 ; 1.14) \quad 1.03(0.89 ; 1.20)$

$1.49(1.18 ; 1.88) \quad 1.52(1.15 ; 1.99) \quad 0.98(0.70 ; 1.38)$

$1.97(1.74 ; 2.22) \quad 1.61(1.47 ; 1.76) \quad 1.22(1.05 ; 1.42)$

$0.97(0.86 ; 1.09) \quad 0.98(0.89 ; 1.08) \quad 0.99(0.85 ; 1.16)$

$1.01(0.91 ; 1.13) \quad 0.95(0.87 ; 1.03) \quad 1.07(0.93 ; 1.23)$

$0.99(0.89 ; 1.09) \quad 1.03(0.94 ; 1.12) \quad 0.96(0.85 ; 1.09)$

$1.15(1.02 ; 1.28) \quad 1.15(1.06 ; 1.26) \quad 0.99(0.87 ; 1.13)$

$1.30(1.16 ; 1.46) \quad 1.08(0.98 ; 1.19) \quad 1.21(1.04 ; 1.40)$

$1.08(0.94 ; 1.24) \quad 0.93(0.83 ; 1.04) \quad 1.16(0.99 ; 1.37)$

$1.24(1.19 ; 1.30) \quad 1.11(1.02 ; 1.20) \quad 1.12(1.03 ; 1.23)$ 